\title{
Note
}

\section{Decomposition of Soybean Spherosomes by Soybean Phospholipase D}

\author{
Yoriko NakaYama and Makoto Kito \\ Research Institute for Food Science, \\ Kyoto University, Uji, Kyoto 611, Japan \\ Received April 7, 1981
}

Soybean oils and proteins are stored in sacks of biological membranes. Phospholipids of these membraries are degraded to phosphatidic acid during storage. ${ }^{1)}$ It would be interesting to confirm the assumption that phospholipase D causes such degradation by catalyzing the decomposition of soybean spherosomal membrane.

Isolation of soybean spherosomes were carried out by the method of Jacks et al. ${ }^{2)}$ After centrifugation the floating layers were dialyzed for 2 days against distilled water at $4^{\circ} \mathrm{C}$. This isolated spherosomes were examined on a light microscope Olympus $\mathrm{BH}$ using oil immersion technique (Fig. 1). The spherosomes were $1 \sim 2 \mu \mathrm{m}$ in diameter. Lipids were analyzed as described previously." The ratio of phospholipids to triacylglycerol in the spherosomes was 0.008 . This value seems to the amount required to enclose the spherosome within a phospholipid

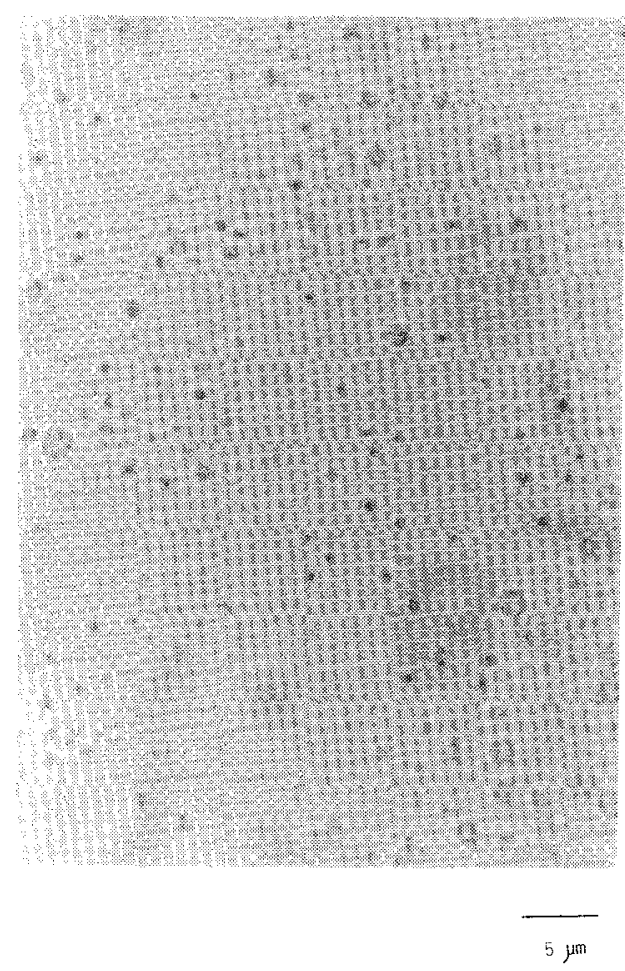

Fig. 1. Light Micrograph of Isolated Spherosomes $(\times 1000)$.

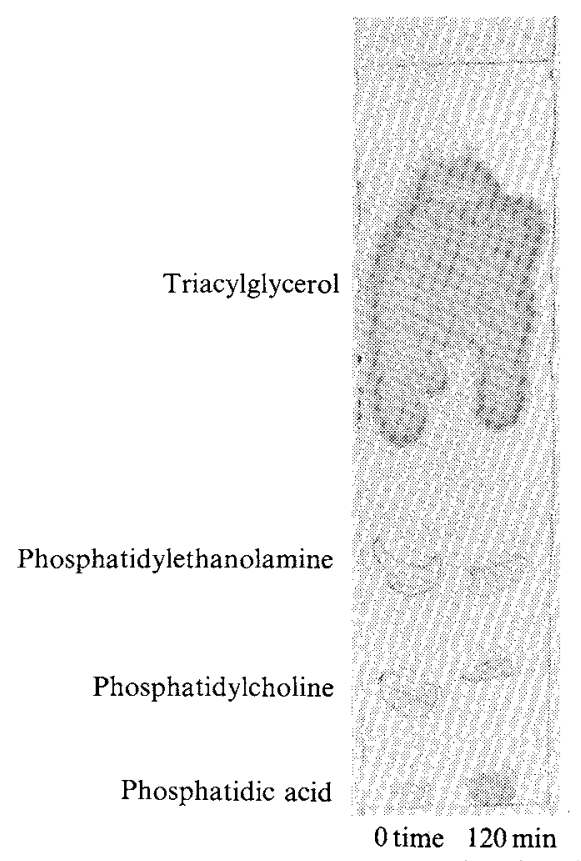

FIG. 2. Digestion of Spherosomal Phospholipids by Phospholipase D. 


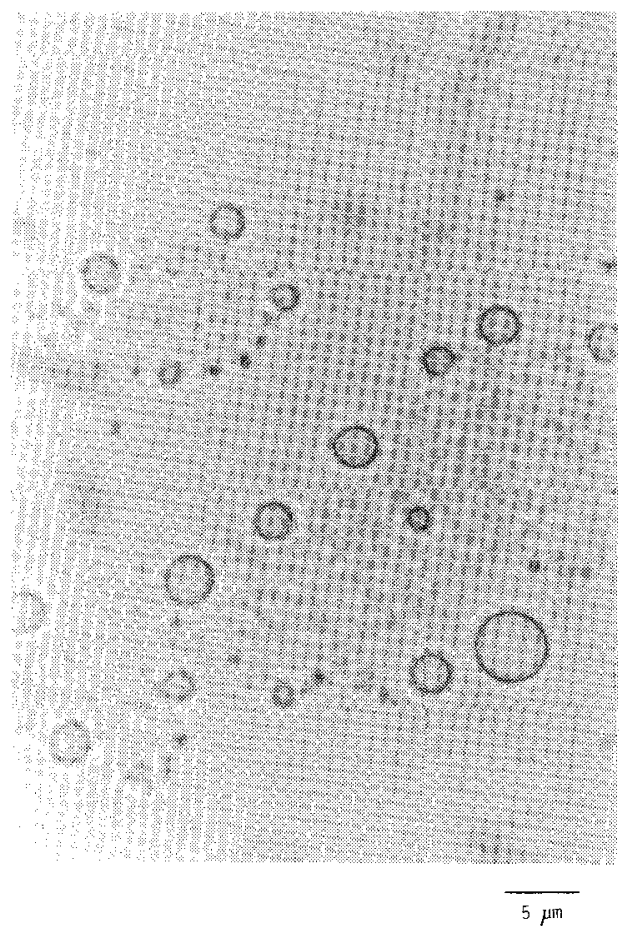

FIG. 3. Light Micrograph of Isolated Spherosomes after Phospholipase D Treatment $(\times 1000)$.

monolayer according to Cosmo et al. ${ }^{3)}$ The spherosome phospholipids consisted of phosphatidylcholine $(67.5 \%)$, phosphatidylethanolamine $(20.0 \%)$, phosphatidylinositol $(5.9 \%)$ and phosphatidic acid $(6.6 \%)$. This proportion of phosphatidylcholine was relatively high compared to that in whole soybeans.

Isolated spherosomes were incubated for $2 \mathrm{hr}$ at $35^{\circ} \mathrm{C}$ with phospholipase $\mathrm{D}$ preparation which had been precipitated in $35 \%$ saturation with ammonium sulfate from the extract obtained from soybean with $10 \mathrm{~mm}$ acetate buffer, $\mathrm{pH}$ 5.0. The reaction was terminated by adding of ethylenediaminetetraacetic acid to a final concentration of $15 \mathrm{~mm}$. The phospholipids which were subsequently extracted with chloroform-methanol $(2: 1 \mathrm{v} / \mathrm{v})$ were analyzed by thin-layer chromatography. ${ }^{4}$ ) Phosphatidylcholine and phosphatidylethanolamine were found to be decomposed with the concomitant increase in phosphatidic acid (Fig. 2). The time-dependent formation of phosphatidic acid was observed in this reaction (data not shown). When the reaction mixture were observed by light microscopy (Fig. 3), small particles were observed in the spherosome preparation at 0 time, whereas larger spherical structures increased after the reaction. This suggests that the resulting of oil droplets may form bigger droplets. Degradation of phospholipids appears to bring about disintegration of organelle membranes, and as a result, the oils in spherosomes are exposed to the cytoplasm and undergo deterioration.

\section{REFERENCES}

1) Y. Nakayama, K. Saio, M. Kito, Cereal Chem., in Press.

2) T. J. Jacks, L. Y. Yatsu and A.M. Altschul, Plant Physiol., 42, 585 (1967).

3) G. M. Cosmo, B. M. Julia, K. R. Oscar and E. P. Delbert, Biochem., 5, 1454 (1966).

4) M. Nishihara and M. Kito, Biochim. Biophys. Acta, 531, 25 (1978). 